\title{
Development of Surgical Tools and Procedures for Experimental Preclinical Surgery Using Computer Simulations And 3D Printing
}

\author{
https://doi.org/10.3991/ijoe.v16i09.15183 \\ Jan Barcik $(\varpi)$ \\ AO Research Institute Davos, Davos, Switzerland \\ Bulgarian Academy of Sciences, Institute of Metal Science 'Acad. A. Balevski', \\ Sofia, Bulgaria \\ jan.barcikeaofoundation.org \\ Manuela Ernst, Ronald Schwyn, Linda Freitag \\ AO Research Institute Davos, Davos, Switzerland \\ Constantin Edmond Dlaska \\ Orthopedic Research Institute of Queensland, Townsville, Australia \\ Ludmil Drenchev, Stoil Todorov, Hristo Skulev \\ Bulgarian Academy of Sciences, Institute of Metal Science 'Acad. \\ A. Balevski', Sofia, Bulgaria \\ Devakara R. Epari \\ Queensland University of Technology, Brisbane, Australia \\ Stephan Zeiter, Boyko Gueorguiev \\ AO Research Institute Davos, Davos, Switzerland
}

\begin{abstract}
Experimental preclinical surgeries require high accuracy and repeatability in all applied procedures. For in vivo studies investigating bone fracture healing it is crucial to create precisely and consistently the experimental bone fractures in all included subjects. In large animal models the required precision is usually achieved by application of dedicated drilling jigs and cutting guides that often need to be individually designed in order to fulfil the requirements of a particular experiment. Moreover, their final design may considerably differ from the prototypes. Hence, the designing and testing processes require multiple iterative refinement loops substantially increasing the costs of the experiment. Therefore, a framework for development of tailor-made instruments for experimental preclinical surgeries is established to reduce the investment of time and financial effort.
\end{abstract}

Keywords - 3D printing, rapid prototyping, experimental surgery, virtual surgery, bone fractures. 


\section{Introduction}

Decades of in vivo preclinical experiments have provided experimental data shaping the current understanding of bone biology and bone fracture healing [1]. Many clinical applications of orthopaedic implants were preceded by extensive preclinical trials [2, 3] to validate new technologies in the in vivo settings and indicate directions for development of new therapies related to bone fracture healing.

To safeguard animal welfare during in vivo trials, the $3 \mathrm{R}$ (replace-reduce-refine) principle was established [4] and is currently widely accepted as an international standard in preclinical research. According to it, the following guidelines should be adopted while designing a preclinical study:

- Reduce the number of involved animals to a minimum

- Refine the procedures to make sure that animal suffering is minimized

- Replace the use of animals with alternative research tools

To comply with the $3 \mathrm{R}$ principle, all surgical procedures in the preclinical research have to be reproduceable and highly standardized. In studies related to bone fracture healing, it is particularly important to create the experimental fractures precisely, consistently and repeatably among all animals included in the study. In large animal models (e.g., sheep, minipig), experimental fractures are most often created by means of osteotomies - bone cuts performed with an oscillating or Gigli saw [5]. Besides the required precision in fracture creation, it is crucial to fix consistently the bone fragments, thereby assuring the same local biomechanical conditions in the fracture gap [6] for all subjects within each study group.

The required precision and repeatability are usually achieved with the use of various drilling jigs and cutting guides. Cutting guides ensure setting of the experimental fractures with the right location and size as required in the preoperative plan. Drilling jigs enable precise positioning of the orthopaedic screws and pins used for fracture fixation.

To reduce the number of animals involved in the experiment, all confounding factors influencing the fracture healing progression should be minimized. Whereases the natural differences between animals (e.g., weight, age, activity) which may influence the study results cannot be avoided, the experimental procedures can be highly standardized to obtain as homogeneous conditions for healing as possible among all animals.

If the experimental fractures are not created with dedicated tools, the unavoidable differences between them will lead to nonhomogeneous local biomechanical conditions in the fracture gap. For example, a planned transverse osteotomy may become slightly oblique, thereby introducing additional forces in the fracture gap with uncontrollable influence on the healing progression between the animals.

The proper design of cutting guides and drilling jigs is therefore crucial for the success of the entire preclinical study. In case of experimental fractures being not comparable between all included animals, the interpretation of the study results becomes difficult and might impair their impact. 
In accordance with the design of a particular in vivo study, the used surgical jigs often need to be individually designed to fulfil specific requirements. Moreover, the final design of a surgical tool is usually preceded by several prototyping steps evolving to it through multiple iteration loops. Figure 1a depicts all main consecutive stages of a typical process during the development and production of tailor-made tools for preclinical surgeries. First, an appropriate animal model, a fracture model and a fixation method are selected, followed by design of the surgical procedure, constituting the initial requirements for the necessary surgical equipment. Second, tailor-made equipment is developed and produced to facilitate the surgical procedure and ensure its repeatability. Third, prior to in vivo application, the new tool is tested during a cadaveric mock surgery. If the latter reveals unforeseen feasibility issues being not anticipated, the design of the surgical procedure and/or the design of the tool must be refined by recursive loops.

It is widely known that the costs of design changes increase with each consecutive stage of product development $[7,8]$. This also applies to modification of tailor-made equipment for preclinical surgeries if changes have to be made after production and testing of the equipment on cadaveric samples (Figure 1b).

Therefore, to reduce such costs, we have introduced a framework enabling to conduct most of the necessary tests during the equipment development phase, and thereby minimising the risk of required design changes later. Virtual simulation approach and additive manufacturing technology are incorporated in the process of designing tailor-made surgical tools to perform all required tests along with equipment development.

(a)
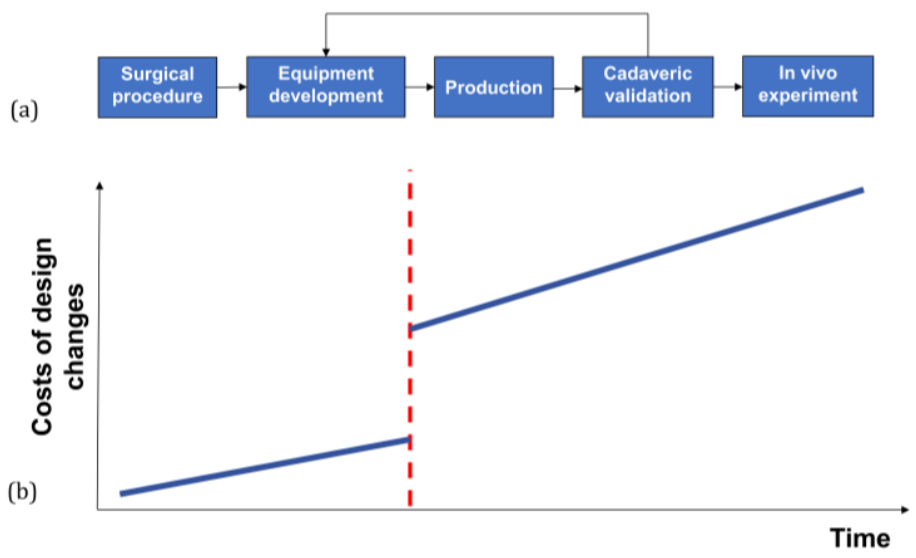

Fig. 1. Stages of the workflow for development and production of tailor-made surgical equipment (a), together with its costs for the design changes (b). Costs for design modifications increase substantially when production stage is entered. In case of unsuccessful cadaveric validation, the return to equipment development stage causes substantial increase in costs.

Based on computed tomography (CT) or magnetic resonance imaging (MRI) data [9], three-dimensional (3D) computer models of bones and joints are already used to 
mimic orthopaedic surgical procedures [10-12] and provide a basis to design patientspecific instruments, e.g., for total knee arthroplasty [13] or resection of bone tumours [14]. In addition, the 3D printing technology helps in the personalization of surgical tools according to the needs of the individual surgeon [15]. George et al. [16] described an iterative design process where general surgical tools, such as scalpel handles and forceps, can be modified according to surgeons' feedback. Moreover, 3D printing technology is not only applied for preparation of surgical tools but for manufacturing of custom made implants too [17] and is frequently utilized for development and production of craniomaxillofacial (CMF) implants $[18,19]$.

Virtual simulations $[20,21]$ and 3D printing $[22,23]$ substantially accelerate the engineering design process. Therefore, we hypothesized that their incorporation in the development and production of tailor-made surgical tools for experimental preclinical surgeries will substantially reduce investment of time and financial efforts.

\section{Materials and Methods}

\subsection{Design requirements}

The methodology was set and tested during the development of the surgical equipment necessary for implantation of a modified active external fixator described by Tufekci et al. [24], designed for instrumentation in sheep tibia. The fracture model consisted of two defects: a $3 \mathrm{~mm}$ experimental defect and a $30 \mathrm{~mm}$ critical-size defect separated by a $30 \mathrm{~mm}$ long mobile bone fragment (Figure 2a). The proximal part of the tibia was fixed to its distal part with an unilateral external fixator, whereas the mobile fragment was fixed to the proximal part with an active external fixator. The latter was equipped with a linear actuator to move the mobile fragment along the bone axis, thereby compressing the healing tissue in the experimental defect.

It is widely accepted that cyclic compression is beneficial for fracture healing, however, the influence of the fracture's local biomechanical environment on bone healing has not been entirely understood yet [25]. Tufekci's fracture model [24] allows for installation of well-controlled biomechanical conditions in the experimental fracture gap, as it is shielded from the influences of functional loading applied by the animal itself. Therefore, this model enables a detailed investigation of the impact of local biomechanical conditions, acting in the fracture gap, on the fracture healing progression.

Figure 2 illustrates the principle of model's operation and depicts the planes of sawing to create the experimental defect and the directions of drilling to position the surgical screws. The implantation of an active fixation on the double osteotomy model is a complicated and surgically demanding [24] procedure. Malalignment of the experimental defect may introduce nonhomogeneous strain between the fracture fragments. The surgical screws (Schanz screws, DePuy Synthes, Zuchwil, Switzerland) need to be precisely positioned - too little distance between them weakens the bony bridge between the screws and may lead to fractured bone between them. The model requires accurate osteotomies in the predefined planes and screws positioned at 
predefined distances with respect to each other to ensure adequate placement of the active fixator on the sheep tibia.

Therefore, it was necessary to design and manufacture cutting guides and drilling jigs to facilitate this complicated surgical procedure.

The steam sterilisation process at $132^{\circ} \mathrm{C}$ and $106 \mathrm{kPa}$ pressure [26] dictates the requirements for selection of materials used for production of the surgical tools. Moreover, during the sawing procedure, small particles may detach from the cutting guide when the sawing blade hits it. Hence, the material for its manufacturing must not be toxic and harming the animal because of possible contact with physiological fluids in its body for a long time.

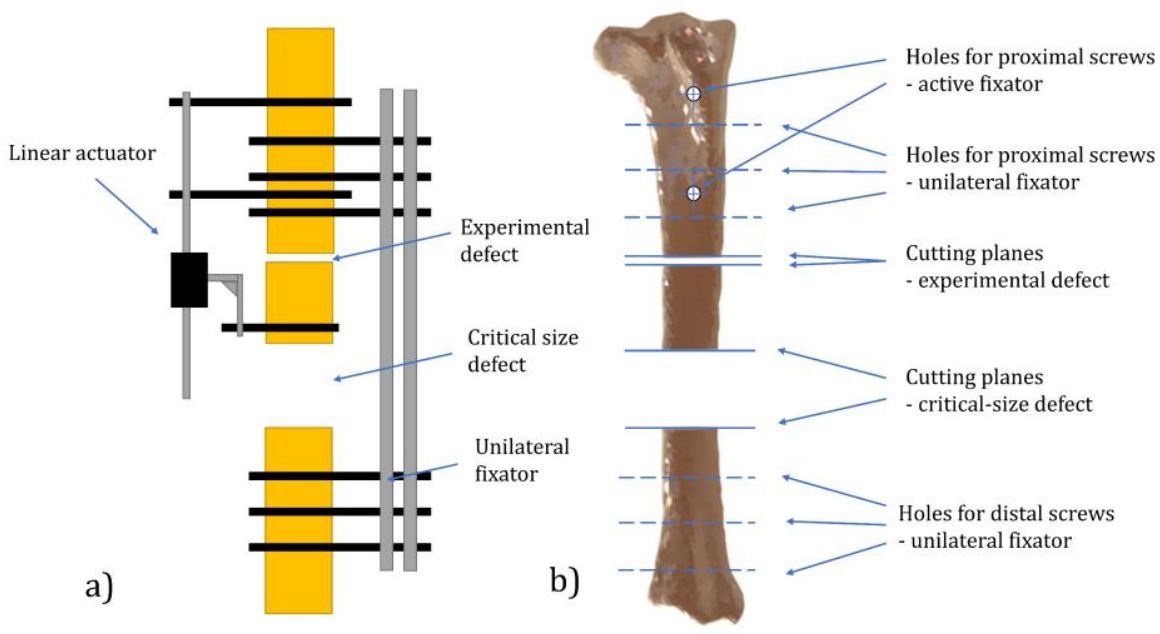

Fig. 2. a) Configuration of the double osteotomy model [24]. The linear actuator, embedded in the active fixator, moves the mobile fragment along the bone axis, thereby compressing the tissue in the experimental defect. Black bars represent the surgical screws. b) Sheep tibia with indicated holes and cutting planes. In the animal experiment, the unilateral and the active fixators are inclined $90^{\circ}$ towards each other, however, for better visualization of their operational principle they are presented at $180^{\circ}$ inclination towards each other in Figure a).

\subsection{Workflow of the developed framework}

Figure 3 depicts the workflow from Figure 1a with added details of the embedded development framework. Based on the requirements originating from the surgical procedure, the first prototypes were designed by computer-aided design (CAD) technology and subsequently virtually tested with the use of SolidWorks software package (Dassault Systèmes, Vélizy-Villacoublay, France) on a 3D model of a sheep tibia. To perform virtual surgery using the prototypes, 3D bone geometry was obtained from a CT scan of a Swiss White Alpine Sheep using a SOMATOM Emotion 6 scanner (Siemens Healthcare GmbH, Erlangen, Germany) and rendered as a 3D model in Amira 
software (Thermo Fisher Scientific, Waltham, USA, Figure 4a). The virtual surgery enabled preliminary loops for refinement of the prototypes (Figure 4b). Subsequently, the prototypes, validated during the virtual surgery, were manufactured by means of 3D printing (Ultimaker B.V., Utrecht, Netherlands) from acrylonitrile butadiene styrene (ABS) filament (Formfutura BV, Nijmegen, The Netherlands, Figure 4c) and tested in a cadaveric mock surgery (Figure 4d). Overall, 4 such surgeries were performed to test the functionality and usability of the prototypes, followed by refinement of their design and $3 \mathrm{D}$ printing for the next test.

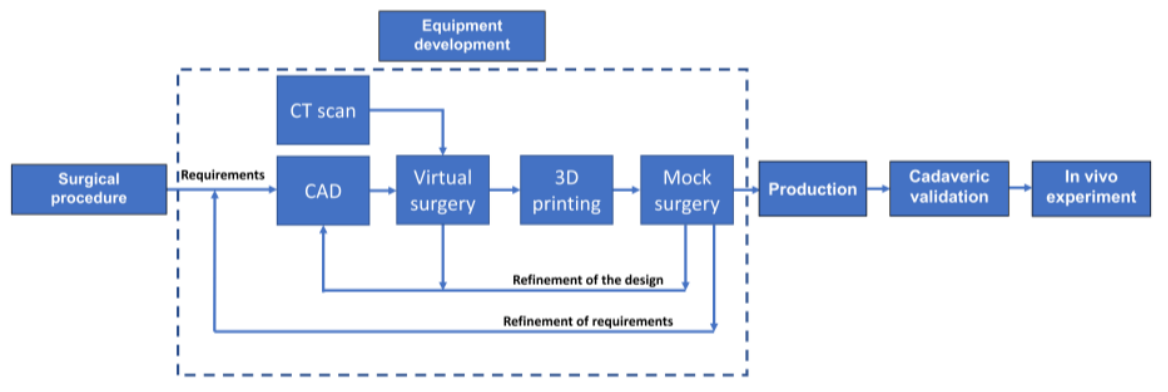

Fig. 3. Workflow for development and production of tailor-made surgical equipment with added details of the embedded development framework (dashed-line frame).

(a)

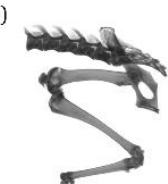

(b)
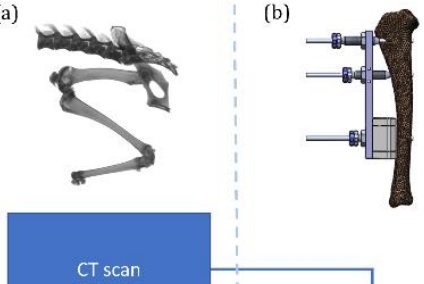

CAD (c)
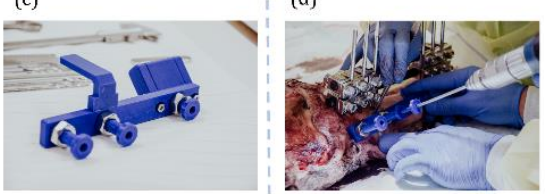

Fig. 4. Four stages of the framework for development of tailor-made tools for experimental preclinical surgery. First, a 3D model of a sheep tibia is generated from a CT scan and CAD models of the prototypes are designed (a). Second, the prototypes are positioned on the bone geometry via a virtual surgery (b). Consequently, the prototypes are 3D printed (c) and tested via a mock surgery (d).

Finally, the tailor-made tools were manufactured from stainless steel 1.4301 by subtractive machining and validated during an additional cadaveric surgery for use in the in vivo experiment. Having an excellent intercrystallite corrosion resistance 
[27], this austenitic stainless steel is used for production of surgical instruments and sanitary installations [28].

Following the surgical procedure validation, the tools were utilized in preclinical surgeries on Swiss White Alpine Sheep (Figure 5). The experiments were approved by the local ethics committee for animal research of the canton of Grisons in Switzerland (approval number TVB 2017_23). The experimental fractures were set under general anaesthesia and appropriate analgesia on right tibiae. The defects were instrumented with an unilateral external fixator and a supplemental active fixator. The correctness of drilling and sawing was verified under fluoroscopic control.

The framework's efficiency in reducing costs and time was assessed by a comparison with the costs and time required for development and production of the tools via conventional methods. The production costs of parts manufactured with conventional methods were estimated using the Solidworks costing utility [29], assuming that the material for prototypes is Aluminium 6061. The costs to produce prototypes via 3D printing and the time required were assessed using the material consumption and manufacturing time predicted by slicer software (CURA 4.2.1., Ultimaker B.V., Utrecht, Netherlands). The total production cost included material costs, costs of power consumption, and labour costs of operating the printer (15 minutes per print according to [30]). Depreciation costs of the printer were not included.

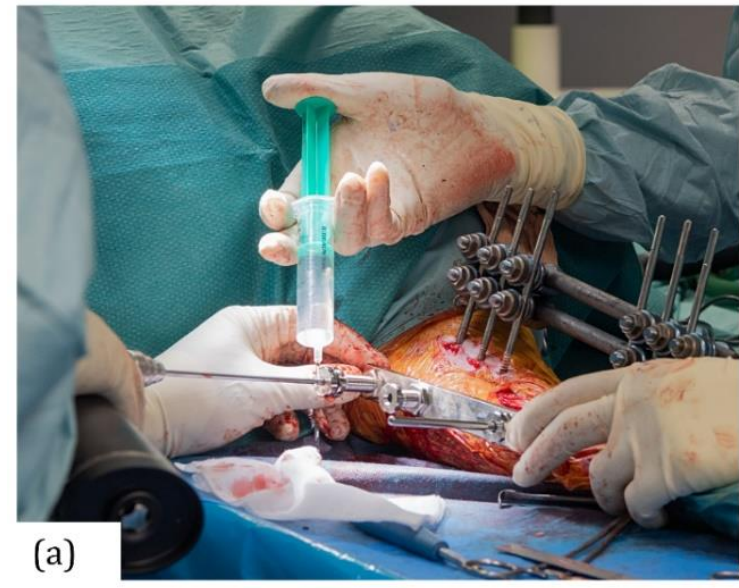

Drilling procedure

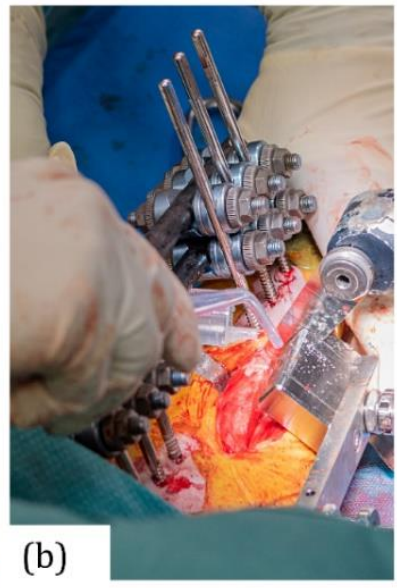

Sawing procedure

Fig. 5. In vivo application of the tailor-made tools

\section{$3 \quad$ Results}

During the iterative application of the workflow described above, the design of the tools changed considerably. Initially, we manufactured and used a prototype, consisting of a plate and 3 columns with special sleeves to guide the insertion of both surgical drill 
bits and orthopaedic Schanz screws with $4.3 \mathrm{~mm}$ and $5.0 \mathrm{~mm}$ diameters, respectively (Figure 6a). Then, in our effort to optimize the surgical procedure, we assumed that the jig would be needed for drilling only and the screws would properly follow the drilled holes orientation without any guidance. Therefore, the sleeves were neglected in the second prototype (Figure 6b). Moreover, we realized that for precise screw positioning each guiding column must be settled firmly on the bone surface with the plate of the jig oriented parallel to the bone axis regardless of the tibia curvature. Therefore, the guiding columns were threaded and secured with nuts (Figure 6c). Finally, it was observed that the screws, inserted without guidance, were not properly oriented, and the sleeves were introduced again in the ultimate design which also incorporated a cutting block to create the critical-size defect (Figure 6d).

(a)

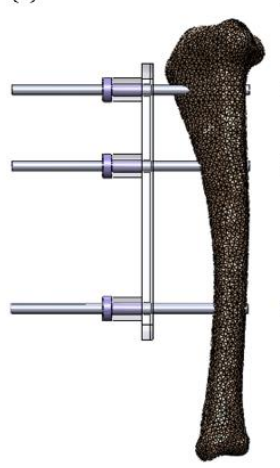

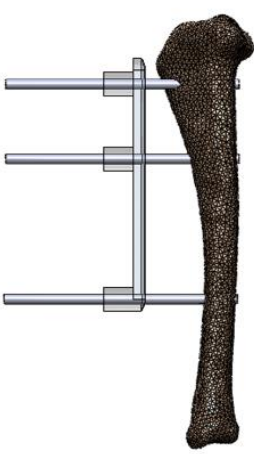

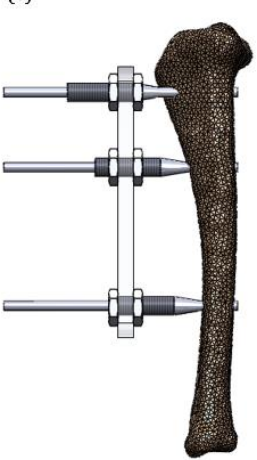

(d)

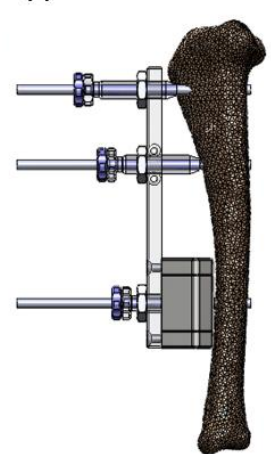

Fig. 6. Stages of the drilling jig and cutting guide development - visualization with drill bits inserted in a tibia model. a) Primary version of a prototype with sleeves for coaxiality of drill bits and screws. b) Second prototype version without sleeves. c) Third prototype version with threaded guiding columns. d) Final prototype design with threaded guided columns, coaxial sleeves and cutting block to guide a saw for creation of a critical-size defect.

The developed tools were successfully applied during preclinical implantations of an active fixator in a sheep model. The Schanz screws were accurately placed with the guidance of the drilling jig and the experimental fracture was adequately set (Figure 7). The developed tools considerably facilitated the surgical procedure, achieving highly accurate implant positioning. No complications related to the tailor-made surgical equipment were observed during the surgeries and all animals recovered well from the surgery. 

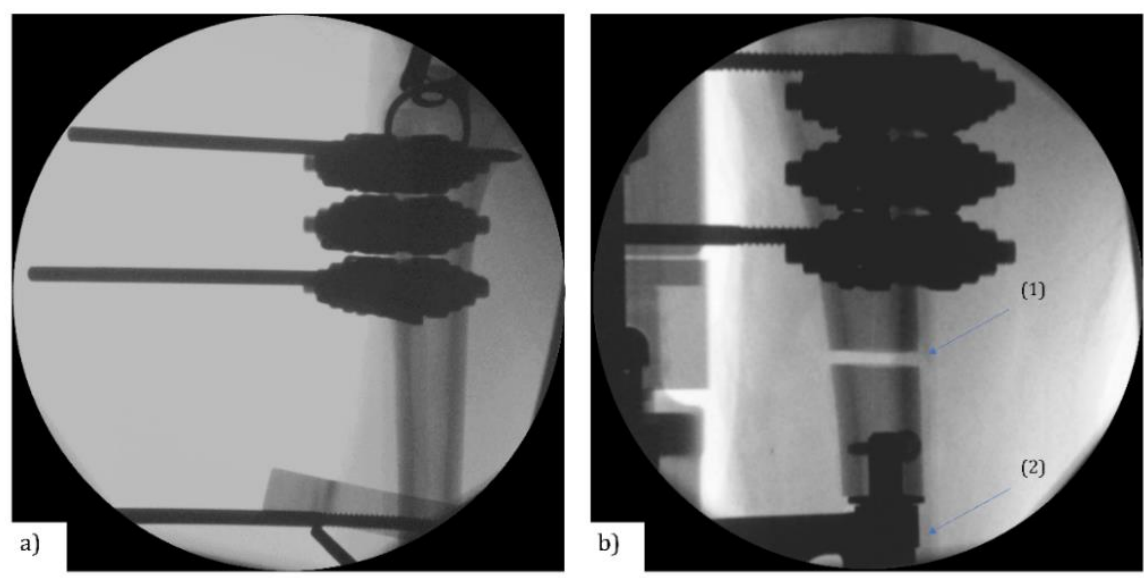

Fig. 7. Intraoperative X-ray images. a) Precise and parallel positioning of the screws for attachment of the active fixator; b) Experimental (1) and critical-size (2) defects created precisely.

Table 1 summarizes the number of design iterations together with evaluation of the costs and time for development and production of the 4 tailored-made prototypes applying both conventional methods and the framework via 3D printing described in this work. The latter enabled development and production of the tools with only $5.5 \%$ of the financial resources compared to conventional methods. The time required for development and production of the prototypes was shortened 3.8 times. The cost for development and production of each prototype via 3D printing was approximately 16 USD, with its major parts being for preparation of the model and operation of the printer.

Table 1. Estimated costs and time for development and production of the 4 prototypes.

\begin{tabular}{|c|c|c|c|c|c|}
\hline \multirow[b]{2}{*}{ Iteration loop } & \multirow[b]{2}{*}{ Prototype } & \multicolumn{2}{|c|}{ Via conventional methods } & \multicolumn{2}{|c|}{ Via 3D printing } \\
\hline & & Cost [USD] & $\begin{array}{c}\text { Time } \\
\text { [h:min:sec] }\end{array}$ & Cost [USD] & $\begin{array}{c}\text { Time } \\
\text { [h:min:sec] }\end{array}$ \\
\hline 1 & 1 & 144.61 & 4:44:00 & 15.75 & 2:00:00 \\
\hline 2 & 2 & 99.28 & $3: 17: 00$ & 15.40 & 1:03:00 \\
\hline 3 & 3 & 174.74 & $5: 47: 00$ & 15.79 & 2:09:00 \\
\hline 4 & Final & 772.81 & $25: 02: 00$ & 16.90 & 4:54:00 \\
\hline Total & & 1191.44 & $38: 50: 00$ & 63.84 & 10:06:00 \\
\hline
\end{tabular}

\section{Conclusions and Summary}

Preclinical experiments are an essential part of research and development in orthopaedics and traumatology. To minimize the number of animals involved in the experiments according to the $3 \mathrm{R}$ principle, uncontrollable confounding factors that may influence the study outcomes should be excluded, assuring repeatability in the creation of experimental bone fractures and fixation procedures. The necessary precision is 
usually achieved with application of cutting guides and drilling jigs. However, they need to be individually tailored to the particular study.

We have set up a framework for development and production of tailor-made tools for experimental preclinical surgeries allowing a substantial decrease in development costs and time, while assuring precise and repeatable conduction of the surgical procedures. The application of this framework facilitated the collaboration with surgeons, allowing hands-on experience with the tools and prompt implementation of the surgeons' feedback.

The framework was implemented during the development and production of the surgical tools required for the precise implantation of an active fixator in a sheep double osteotomy model. Even though the use of a double osteotomy model with an unilateral and an active fixator is described in the literature [24], the changes in the design of the active fixator, introduced in the current work, caused substantial modifications of the surgical procedure. Computer simulations enhanced critical evaluation of the latter by virtual positioning of the fixators and testing of the required tools on a $3 \mathrm{D}$ model of a sheep tibia. The 3D printing technology enabled multiple testing of the procedure on cadaver specimens, resulting in no surgical complications during the in vivo experiment.

In the current work, we created and successfully tested a framework for development and production of tailor-made tools for experimental preclinical surgery and demonstrated its capabilities in terms of considerably decreased costs and time. The framework can be applied in further preclinical studies requiring preparation of dedicated tools to facilitate surgical procedures.

\section{Acknowledgement}

The authors are not compensated and there are no other institutional subsidies, corporate affiliations or funding sources supporting this work unless clearly documented and disclosed. This study was performed with the assistance of the AO Foundation via the AOTRAUMA Network (Grant No.: AR2016_06).

\section{$6 \quad$ References}

[1] Y. H. An and R. J. Friedman, Eds. Animal models in orthopaedic research. Boca Raton: CRC Press, 1999.

[2] M. Allgöwer, S. Perren, and P. Matter, "A new plate for internal fixation - The dynamic compression plate (DCP)," Injury, vol. 2, no. 1, pp. 40-47, 19701970. https://doi.org/10.1016/s0020-1383(70)80111-5

[3] S. M. Perren et al., "The concept of biological plating using the limited contact-dynamic compression plate (LC-DCP). Scientific background, design and application" Injury, Conference paper vol. 22, no. Suppl 1, pp. 1-41, 1991 1991. https://doi.org/10.1016/00201383(91)90123-v

[4] W. M. S. Russell and R. L. Burch, The principles of humane experimental technique. Methuen, 1959. 
[5] S. Decker, M. O. Janin Reifenrath, C. Krettek, and C. W. Müller, "Non-osteotomy and osteotomy large animal fracture models in orthopedic trauma research," Orthopedic reviews, vol. 6, no. 4, 2014. https://doi.org/10.4081/or.2014.5575

[6] L. E. Claes et al., "Effects of mechanical factors on the fracture healing process," Clin Orthop Relat Res, no. 355 Suppl, pp. S132-47, Oct 1998.

[7] Boehm, "Software Engineering," IEEE Transactions on Computers, vol. C-25, no. 12, pp. 1226-1241, 1976.

[8] P. MacLeamy, "Collaboration, integrated information and the project lifecycle in building design, construction and operation," WP-1202, The Construction Users Roundtable, 2004.

[9] O.-R. Kwon, K.-T. Kang, J. Son, Y.-J. Choi, D.-S. Suh, and Y.-G. Koh, "The effect of femoral cutting guide design improvements for patient-specific instruments," BioMed Research International, vol. 2015, 2015. https://doi.org/10.1155/2015/978686

[10] S. Klein, C. M. Whyne, R. Rush, and H. J. Ginsberg, "CT-based patient-specific simulation software for pedicle screw insertion," Clinical Spine Surgery, vol. 22, no. 7, pp. 502-506, 2009. https://doi.org/10.1097/bsd.0b013e31819877fd

[11] A. S. Rose, C. E. Webster, O. L. Harrysson, E. J. Formeister, R. B. Rawal, and C. E. Iseli, "Pre-operative simulation of pediatric mastoid surgery with 3D-printed temporal bone models," International journal of pediatric otorhinolaryngology, vol. 79, no. 5, pp. 740-744, 2015. https://doi.org/10.1016/j.ijporl.2015.03.004

[12] J. P. Torres, "Virtual Reality Applied to Dental Implant Surgery," International Journal of Online Engineering, vol. 8, no. 1, 2012.

[13] G. G. Jones, S. Clarke, M. Jaere, and J. Cobb, "3D printing and unicompartmental knee arthroplasty," EFORT open reviews, vol. 3, no. 5, pp. 248-253, 2018. https://doi.org/10. $\underline{1302 / 2058-5241.3 .180001}$

[14] L. Ma et al., "3D-printed guiding templates for improved osteosarcoma resection," Scientific reports, vol. 6, p. 23335, 2016.

[15] S. Kondor et al., "Personalized surgical instruments," Journal of Medical Devices, vol. 7, no. 3, 2013.

[16] M. George, K. R. Aroom, H. G. Hawes, B. S. Gill, and J. Love, "3D printed surgical instruments: the design and fabrication process," World journal of surgery, vol. 41, no. 1, pp. 314-319, 2017. https://doi.org/10.1007/s00268-016-3814-5

[17] L. Ma et al., "3D printed personalized titanium plates improve clinical outcome in microwave ablation of bone tumors around the knee," Scientific reports, vol. 7, no. 1, pp. 110, 2017. https://doi.org/10.1038/s41598-017-07243-3

[18] J. Ma, L. Ma, Z. Wang, X. Zhu, and W. Wang, "The use of 3D-printed titanium mesh tray in treating complex comminuted mandibular fractures: a case report," Medicine, vol. 96, no. 27, 2017. https://doi.org/10.1097/md.0000000000007250

[19] L. Gaviria, J. J. Pearson, S. A. Montelongo, T. Guda, and J. L. Ong, "Three-dimensional printing for craniomaxillofacial regeneration," Journal of the Korean Association of Oral and Maxillofacial Surgeons, vol. 43, no. 5, pp. 288-298, 2017. https://doi.org/10. 5125/jkaoms.2017.43.5.288

[20] M. Dodgson, D. M. Gann, and A. Salter, "The impact of modelling and simulation technology on engineering problem solving," Technology Analysis \& Strategic Management, vol. 19, no. 4, pp. 471-489, 2007. https://doi.org/10.1080/ $\underline{09537320701403425}$

[21] P. Garaizar, M. Vadillo, and D. Lopez-de-Ipina, "Benefits and pitfalls of using HTML5 APIs for online experiments and simulations," in 2012 9th International Conference on Remote Engineering and Virtual Instrumentation (REV), 2012, pp. 1-7: IEEE. https://doi.org/10.1109/rev.2012.6293120 
[22] M. Attaran, "The rise of 3-D printing: The advantages of additive manufacturing over traditional manufacturing," Business Horizons, vol. 60, no. 5, pp. 677-688, 2017. https://doi.org/10.1016/j.bushor.2017.05.011

[23] A. Bacha, A. H. Sabry, and J. Benhra, "Fault Diagnosis in the Field of Additive Manufacturing (3D Printing) Using Bayesian Networks," International Journal of Online and Biomedical Engineering (iJOE), vol. 15, no. 03, pp. 110-123, 2019. https://doi.org/10.3991/ijoe.v15i03.9375

[24] P. Tufekci et al., "Early mechanical stimulation only permits timely bone healing in sheep," Journal of Orthopaedic Research, vol. 36, no. 6, pp. 1790-1796, 2018/06/01 2018. https://doi.org/10.1002/jor.23812

[25] V. Glatt, C. H. Evans, and K. Tetsworth, "A Concert between Biology and Biomechanics: The Influence of the Mechanical Environment on Bone Healing," Frontiers in physiology, vol. 7, pp. 678-678, 2017. https://doi.org/10.3389/fphys.2016.00678

[26] S. Lerouge, "Sterilization and cleaning of metallic biomaterials," in Metals for Biomedical Devices: Elsevier, 2019, pp. 405-428. https://doi.org/10.1016/b978-0-08-102666-3.00016-x

[27] "Steel 1.4301," L. MATTHEY, Ed., ed, 2013.

[28] R. Dubovská, J. Majerík, R. Čep, and K. Kouřil, "Investigating the influence of cutting speed on the tool life of a cutting insert while cutting DIN 1.4301 steel," 2016. https://doi.org/10.17222/mit.2015.036

[29] D. LUKIĆ, M. M.-S. B.-M. ĐURĐEV, and J. V.-A. ANTIĆ, "Manufacturing cost estimation in the conceptual process planning," planning, vol. 30, p. 50, 2016.

[30] The Swiss Federal Statistical Office. (2016, 24.03.2020). Labour costs Switzerland. https://www.bfs.admin.ch/bfs/en/home/statistics/work-income/wages-incomeemployment-labour-costs/labour-costs.html https://doi.org/10.1787/888932786593

\section{$7 \quad$ Authors}

Jan Barcik is a PhD student at the Biomedical Development Program, AO Research Institute Davos, Davos, Switzerland and at the Institute of Metal Science 'Acad. A. Balevski', Bulgarian Academy of Sciences, Sofia, Bulgaria.

Manuela Ernst is a Project Leader at the Biomedical Development Program, AO Research Institute Davos, Davos, Switzerland.

Ronald Schwyn is a Project Leader at the Biomedical Development Program, AO Research Institute Davos, Davos, Switzerland.

Linda Freitag Dr. med. vet., is a Project Leader at the Preclinical Services Program, AO Research Institute Davos, Davos, Switzerland.

Constantin Edmond Dlaska, Dr. med. univ. FEBOT, FaOrtho, is an Orthopaedic and Traumatology Surgeon at the Orthopaedic Research Institute of Queensland, Townsville and works at the Mackay Base Hospital, Queensland, Australia.

Professor Ludmil Drenchev, PhD, DSc, is Director of the Institute of Metal Science 'Acad. A. Balevski', Bulgarian Academy of Sciences, Sofia, Bulgaria.

Professor Stoil Todorov, PhD, is Head of the Department of Mechanical Devices, Equipment and Technologies, Institute of Metal Science 'Acad. A. Balevski', Bulgarian Academy of Sciences, Sofia, Bulgaria.

Professor Hristo Skulev, PhD, DSc, is Expert Education and Science at the Institute of Metal Science 'Acad. A. Balevski', Bulgarian Academy of Sciences, Sofia, Bulgaria. 
Professor Devakara R. Epari, PhD, is an Associate Professor at the Institute of Health and Biomedical Innovation at the Queensland University of Technology, Brisbane, Australia.

Stephan Zeiter, PhD, Dr. med. vet., Dipl ECLAM, is Program Leader at the Preclinical Services Program, AO Research Institute Davos, Davos, Switzerland.

Professor Boyko Gueorguiev, $\mathrm{PhD}$, is Program Leader at the Biomedical Development Program, AO Research Institute Davos, Davos, Switzerland.

Article submitted 2020-04-24. Resubmitted 2020-05-22. Final acceptance 2020-05-23. Final version published as submitted by the authors. 